\title{
DOOB: A HALF-CENTURY ON
}

\author{
N. H. BINGHAM,* University of Sheffield
}

\begin{abstract}
Probability theory, and its dynamic aspect stochastic process theory, is both a venerable subject, in that its roots go back to the mid-seventeenth century, and a young one, in that its modern formulation happened comparatively recently - well within living memory. The year 2003 marked the seventieth anniversary of Kolmogorov's Grundbegriffe der Wahrscheinlichkeitsrechnung, usually regarded as having inaugurated modern (measuretheoretic) probability theory. It also marked the fiftieth anniversary of Doob's Stochastic Processes. The profound and continuing influence of this classic work prompts the present piece.
\end{abstract}

Keywords: J. L. Doob; stochastic process; martingale; history

2000 Mathematics Subject Classification: Primary 60-03

Secondary 01A60

\section{Pre-Doob}

Before the emergence of measure theory, the machinery needed to handle probability rigorously did not exist. Hardly surprisingly, the subject was not regarded as mathematically respectable by pure mathematicians. The wonder is that so much of lasting value was achieved - witness the work of Markov and Poincaré, for example. Richard von Mises, writing in the 1920s, regarded probability not only as not being fully mathematical, but as not being fully mathematicisable (see Geiringer (1964, pp. 43-49, especially pp. 44, 48-49)).

This view had begun to change even before the Grundbegriffe (Kolmogorov (1933)) - for historical background see, for example, Bingham (2000). Paul Lévy (1886-1971) published the first measure-theoretic book on probability, Calcul des Probabilités (see Lévy (1925)), following this with his two enduring classics, Théorie de l'Addition des Variables Aléatoires (Lévy (1937)) and Processus Stochastiques et Mouvement Brownien (Lévy (1948)). In his papers, Lévy made profound contributions, not only to limit theorems (the enduring theme of his life's work was the central limit problem and, in particular, limiting Gaussianity in the most natural and general setting) but also to the Lévy-Khinchin formula and to what are now called Lévy processes. See the obituary Loève (1973) for background on Lévy's life and work. It can be observed that Lévy's writing style contrasts strongly with Doob's. Lévy was intuitive ('Alors, ces probabilités sont assez petites...'), and discursive (the discussion ends with what has been proved, in italics - the style of French textbooks of the time, pre-Bourbaki). Doob was careful, formal (theorem followed by proof, in the Landau 'Satz-Beweis' style), and precise.

Partly at the instigation of G. H. Hardy (1877-1947), Harald Cramér (1893-1985) wrote his influential Cambridge tract (Cramér (1937)). With his background in pure mathematics (originally analytic number theory), Cramér was able to take up the lead of the Grundbegriffe

Received 30 July 2003; revision received 23 June 2004.

* Postal address: Department of Probability and Statistics, University of Sheffield, Sheffield S3 7RH, UK.

Email address: nick.bingham@sheffield.ac.uk 
and present, at greater length, a synthesis of much of the subject as it then stood. The title of the present piece is, in part, a tribute to Cramér (1976).

Also coming to probability from a background in analysis was William Feller (1906-1970). Feller began to write on probability in 1935. His deepest work was on the interplay between Markov processes on the one hand and second-order linear differential operators on the other; in particular, questions of classification and boundary conditions. The first edition of his famous book An Introduction to Probability Theory and Its Applications, Vol. 1, appeared in 1950, only three years before Doob's Stochastic Processes, and it is interesting and instructive to compare the two. (Doob's Stochastic Processes will be referred to as 'Doob' below, unless it is clear from context that we mean J. L. Doob the person)

The spectacular career of Wolfgang Döblin (1915-1940), a tragic victim of the Second World War, made a profound impact on the subject (and on Doob's book) in the last three years of his life. For background on the man who ended his life as Vincent Doeblin see, for example, Lindvall (1991) and Cohn (1993). Other important influences were the work of the Russian school (e.g. Kolmogorov and Khinchin), the Polish (e.g. Marcinkiewicz and Zygmund), the Japanese (e.g. Itô), and the American (e.g. Wiener and Stone).

Before embarking on a discussion of Doob, it might be as well to comment on the influence, pre-Doob, of Norbert Wiener (1894-1964). Wiener's greatest contribution to probability and stochastic processes was his 1923 construction of the Wiener measure - essentially, the rigorous proof that 'Brownian motion is continuous'. However, in his writings of the early 1930s, on Fourier integrals, generalized harmonic analysis, Tauberian theory, etc. (and in the 1940s on time series - particularly spectral aspects - and prediction), Wiener convincingly demonstrated the power of Fourier analysis, rigorously and solidly based on the Lebesgue integral, Lebesgue measure, and measure theory. This played an important role in convincing the mathematical public - applied workers as well as theoreticians - that the investment of learning measure theory had to be undertaken. The resulting sea-change in attitude within the profession helped to prepare the ground for Doob.

Doob's book was in fact planned to be a collaborative work with Wiener. To quote the penultimate paragraph of the preface:

Chapter XII, on prediction theory, is somewhat out of place in the book, since it discusses a rather specialized problem. It was put in because of the importance of the subject matter, and because of the lack of material on prediction theory, in the usual language of probability, readily available to the American reader. I had the benefit of stimulating conversations with Norbert Wiener on this subject.

For background on Wiener see, for example, his Collected Works (Wiener (1976), (1979), (1981), (1985)), the biography Masani (1990), and its review Doob (1990).

\section{Doob}

Joseph Leonard Doob (1910-2004) was originally a pure mathematician and analyst, like several of the probabilists already mentioned. His early interests lay in complex analysis and potential theory. Confronted as a young man by the great difficulty of finding an academic job during the Great Depression and its aftermath, he took the advice of Harold Hotelling and decided to specialize in probability and statistics. Doob's book cites thirteen of his papers, written in the period 1934-1951.

The unchallengeable nature of measure theory as the essential mathematical language for probability, and Doob's forthright attitudes and style, are both well exemplified by two sentences 
from the third paragraph of the preface to Doob: 'There has been no compromise with the mathematics of probability. Probability is simply a branch of measure theory, with its own special emphasis and field of application, and no attempt has been made to sugar-coat this fact'.

The many-sided nature of probability and stochastics - as between pure and applied aspects, as between mathematics and statistics, etc. - is also well exemplified by a sentence from Doob's next paragraph:

There is probably no mathematical subject which shares with probability the features that on the one hand many of its most elementary theorems are based on rather deep mathematics, and that on the other hand many of the most advanced theorems are known and understood by many (statisticians and others) without the mathematical background to understand their proofs.

Inescapably, the first problem facing the author of a book on probability or stochastic processes remains what it was half a century ago: how to handle measure theory. (The present writer felt an echo of this when examining recently in the University of Cambridge; both measure theory and stochastic processes are in the curriculum, but the numbers taking the first are tiny compared to those taking the second.) There are basically three ways to proceed. First, a non-measure-theoretic treatment can be presented to the best of the author's ability. Distinguished classics of this kind include Feller (1950); Bartlett (1955) (originally published two years after Doob but conceived, with J. E. Moyal, in 1946); Karlin and Taylor (1975), (1981); and Grimmett and Stirzaker (1982). Second, measure theory and probability can be expounded together (or consecutively in the same book), as in, for example, Kingman and Taylor (1966) or Billingsley (1979). Third, measure theory can be assumed to be known to the reader, referring to a standard book on measure theory as needed - this was the approach taken by Doob. Doob referred to Halmos (1950) (Paul R. Halmos was a pupil of Doob's), but summarises what he needs of measure theory in a 24-page supplement at the end of the book.

The next basic problem concerns the gap between discrete and continuous time. (Readers may care to ask themselves whether time is discrete or continuous. One might reply that how one perceives or experiences time depends on whether one uses a digital watch or one with hands. And of course, how one perceives time affects how one models it.) The essence of measure theory lies in the property of countable additivity. The countability endemic here sits comfortably with the countable setting of discrete time. (The time-set may well be finite, with the setting still having the character of a stochastic process problem rather than one in enumerative combinatorics. This happens in, for example, the binomial tree model of mathematical finance - see Bingham and Kiesel (2004).) However, countable additivity does not sit comfortably with the uncountable time-set encountered in continuous time. Stochastic process theory - the dynamic side of probability, or the mathematics of randomness unfolding with time - will thus, inescapably, always be a subject presenting some difficulty to those who learn it, teach it, or write about it.

Doob began (Chapter I: Introduction and probability background, 45pp.) with a summary of what he needed from probability. Here he treated the Daniell-Kolmogorov theorem (as in the Grundbegriffe), which is essentially the existence theorem for a stochastic process, given the minimal raw material of an appropriately consistent set of finite-dimensional distributions. The main emphasis is on conditioning (Kolmogorov's treatment of conditioning in the Grundbegriffe, using the Radon-Nikodým theorem, being by now well established). He closed with a treatment of characteristic functions, including (with an eye on his intended treatment of infinite divisibility) more on inequalities for them than most readers nowadays will know). 
Doob continued with Chapter II: Definition of a stochastic process - Principal classes (56pp.). The starting point (Section 1) is a consistent set of finite-dimensional distributions, and the Kolmogorov construction from it of a stochastic process (basically, one infinite-dimensional object unifying infinitely many consistent finite-dimensional objects). He addressed (in Section 2) the 'pitfall of uncountability': essentially that, with an uncountable index set (in continuous time, say), the sample paths of the process may lack enough regularity to be tractable. He addressed this with one of his key technical contributions: his theory of separability, measurability, and versions. (In brief - see p. 66 - separability is not a restriction on finite-dimensional distributions, yet measurability is.) The rest of the chapter is a brief introduction to the particular classes of stochastic process to which the remaining ten chapters will be devoted. In particular, the Gaussian section contains - see p. 78 - the intriguing statement ' . . very few facts specifically true of Gaussian processes are known'.

Chapter III (Processes with mutually independent random variables, 46pp.) addressed the core of probability theory, as would be expounded nowadays in a book on probability theory at the measure-theoretic level, but without a specific focus on stochastic processes. It began with zero-one laws. It then treated random series (including, for series with independent terms, the equivalence of almost-sure convergence in probability and in distribution), proved Kolmogorov's strong law of large numbers, treated infinite divisibility, and derived the LévyKhinchin formula.

In Chapter IV (Processes with mutually uncorrelated or orthogonal random variables, 22pp.), the restriction to probability (or even finite) measures is dropped. Versions of the law of large numbers and results on random series are given in this degree of generality. So too, with a view to applications to time series later, are results on the factorization of spectral densities. Doob emphasised that the natural setting here is that of general orthogonal series and, accordingly, referred to analysis books such as Kaczmarz and Steinhaus (1935), Stone (1932), and Zygmund (1935) for proofs.

Doob turned in Chapter IV (Markov processes - Discrete parameter, 64pp.) to Markov chains. Finite chains are treated in some detail. For example, one cannot remain in transient states forever (and so, not all states can be transient). However, persistent (or recurrent) states are not broken down into those that are null and that are positive, as in Feller, and so the nonexistence of null states in finite chains is not given. Card shuffling is discussed (the modern reader will recall Diaconis' result: seven shuffles suffice). General state spaces (see Section 5, pp. 190-218) are treated in one go, with heroic disregard for the frailties of the reader. Topics include Döblin's condition and exponential convergence. Finally, the law of large numbers and central limit theorem are proved, using 'Döblin's trick' (see Chung (1960)).

Markov processes with continuous parameter follow in Chapter VI (57pp.). Finite chains are treated: the $Q$-matrix, holding times, step-function nature of the sample paths, etc. With continuous state space, Döblin's condition is used; the sample paths may have discontinuities worse than jumps. Diffusions are treated in the light of Itô's work (1944-1951) on stochastic integrals and stochastic differential equations.

Doob's Chapter VII on martingales (98pp.) is the most famous in the book. Because it has been so influential, it still has a modern feel to it, apart from terminology (thus filtrations are everywhere, but not under this name; our submartingales and supermartingales appear here as semimartingales and lower-semimartingales, etc.). After the basic definitions (Section 1), Doob turned (Section 2) to games of chance, gambling systems, and the optional stopping theorem. Section 3 (Fundamental inequalities) contains Doob's maximal inequality (generalizing Kolmogorov's, itself generalizing Chebyshev's) and the upcrossing 
inequality. These are applied to convergence theorems in Section 4 (uniformly integrable martingales, martingale convergence, tail sigma-fields, reversed martingales). Sums of independent terms are treated in Section 5 (random series) and Section 6 (the strong law). Integration and differentiation follow in Sections 7 and 8, and likelihood ratios and sequential analysis in Sections 9 and 10. Continuous-parameter martingales are treated in Section 11 (which, at 36 pages, again makes demands on the reader's stamina). In particular, regularization of paths is covered, and Lévy's martingale characterization of Brownian motion is given. The chapter closes (Section 12) with applications of martingale theory to sample path continuity (this is a simple result of Lévy: sample paths of Lévy processes have at worst jump discontinuities).

Chapter VIII (Processes with independent increments, 35pp.) is devoted to (in modern terminology) Lévy processes. Processes with orthogonal increments follow in Chapter IX (27pp.). Stochastic integrals are discussed from the point of view of Wiener and Stone. Application is made to Campbell's theorem (shot noise processes), and to a version of the representation theorem for Brownian martingales.

Stationary processes are treated in discrete (Chapter X, 55pp.) and continuous time (Chapter XI, 53pp.). Here we find the strong law of large numbers, Bochner's theorem, and the mathematics needed for time series in the frequency domain (spectral representation, Wold decomposition, etc.). Linear filtering is also discussed (Chapter XI, Section 9).

The book ends with the admittedly more specialized Chapter XII (Linear least squares prediction - stationary (wide sense) processes, 38pp.). There is a thorough discussion of the 'Kolmogorov-Wiener filter' (linear prediction given the entire past, in the square-integrable case), the Wold decomposition again playing a central role. Both Kolmogorov and Wiener were motivated by wartime problems of fire control, particularly against aircraft. One source here is Wiener's 'Yellow Peril' (Wiener (1949) - based on a version of 1942 with circulation restricted by wartime security). Another is Doob's 40-page Berkeley Symposium paper, Doob (1949).

\section{Post-Doob}

There is general agreement that the two enduring classics of their time are Feller (1950) and Doob. I recall 'Kingman's dictum' from the 1960s and 1970s: 'It's all in Doob' (just as a modern stochastic analyst might say, 'All you need is Itô's lemma' - a dictum I learned from Michael Harrison).

The most obvious successor to Doob, in terms of scope and aims, was perhaps Loève (1955). Revealingly, Loève's book later split into two volumes (see Loève (1977), (1978)). Similarly, Gīhman and Skorohod (1969) split into a three-volume work (Gīhman and Skorohod (1974), (1975), (1979)). As the field continued to develop, it became clear to authors that one could no longer aim to cover everything in stochastic processes in one book of reasonable size. Accordingly, the literature ramified and books on particular kinds of process or particular aspects of the field began to appear. It is interesting to observe the extent to which Doob's book set the research - and textbook - agenda.

Some aspects of stochastic processes are covered in any standard text on probability. Of many such, we first mention Feller (1966). This influential text was planned as part of a three-volume work, with Volume 3 on stochastic processes. Feller's death in 1970 prevented Volume 3 being written. Feller returned repeatedly to what he had already written, revising and polishing, but Doob was content to let his book speak for itself, and eventually 'die quietly on 
the shelf' (personal remark to the author, $c$.1975). Later probability books of comparable level include Breiman (1968), Chung (1968), and Billingsley (1979).

For Markov chains, the standard specialist work remains Chung (1960). For Markov processes, the next generation is dominated by Dynkin (1965), a two-volume work. The role of Döblin's condition is reflected in a number of variants and alternatives, under which Markov processes on general state spaces may be handled, to some extent, in the manner of Markov chains. The best-known such condition is Harris recurrence. For monograph accounts, see Nummelin (1984), Revuz (1984), and Meyn and Tweedie (1993). The role of exponentially fast convergence led to the study of geometric ergodicity by Kendall, Vere-Jones, Pitman, Tweedie, and others.

The theory of infinite divisibility has always been intimately linked with that of processes with (stationary) independent increments. These were previously studied under a variety of unwieldy names, but have been very properly called Lévy processes since at least the early 1970s. The year after Doob appeared, Gnedenko and Kolmogorov (1954) was published in English. Much of this material appears in Fellerian style in Feller (1966). Lévy processes deserve and get a chapter in any serious text on stochastic processes, but received two fine monograph treatments in Bertoin (1996) and Sato (1999) - the first more probabilistic, the second more analytic.

Laws of large numbers received a monograph treatment in Révész (1968), ergodic theorems in Krengel (1985), random series in Kahane (1985), and random walks in Spitzer (1964).

Gaussian processes received a great deal of attention in the Russian school, the French (Fernique (1975)), the American (see, for example, many papers by S. M. Berman and M. B. Marcus) and the Japanese. The textbook literature includes Ibragimov and Rozanov (1978). For further references, developments involving abstract Wiener space, white noise calculus, etc., see Janson (1997).

The literature on time series exploded in the second half of the twentieth century. An early post-Doob book is Whittle (1963). The Kolmogorov-Wiener filter was supplemented by the Kalman filter (just in time for its deployment in its first natural field of application - control of manned spacecraft in real time). For a treatment of filtering and control see, for example, Davis (1977) and, for a contemporary view of time series see, for example, Brockwell and Davis (1987) and the references therein.

Martingales have become ubiquitous since Doob - we live in the age of martingales. They have been studied particularly intensively by D. L. Burkholder, a colleague of Doob's at Illinois. For a fine textbook account, see Neveu (1975); for martingale central limit theory, see Hall and Heyde (1980). Note that Doob covers central limit theory for the Markov chain case, but not the martingale case.

Diffusions in one dimension may be treated by a variety of methods; see, for example, Itô and McKean (1965) and Breiman (1968). In the multidimensional case, there is essentially only one approach, the Stroock-Varadhan approach via martingale problems (Stroock and Varadhan (1979)). For an excellent treatment of much of the material duscussed above, see the two-volume work Rogers and Williams (1994), (1987).

One of the main achievements of Paul-André Meyer (1934-2003) was to develop (particularly following the work of Kunita and Watanabe of 1967 on square-integrable martingales) the theory of stochastic integration; see, for example, Meyer (1976). Meyer's formulation involved predictable integrands and semimartingale integrators (semimartingales in Meyer's sense, not Doob's!). For textbook accounts of the related theories of stochastic integrals and stochastic differential equations see, for example, McKean (1969), Ikeda and Watanabe (1981), 
Øksendal (1985), Protter (1990), Karatzas and Shreve (1988), and Revuz and Yor (1991). A different approach, via 'rough paths', has recently been given by Lyons and Qian (2002).

One topic not covered in Doob (perhaps surprisingly, in view of Doob's background in complex analysis and potential theory) is the link between potential theory and probability; in particular, Markov processes. This goes back to Kakutani (1944), who showed that classical (Newtonian) potential theory is intimately linked with (or corresponds to) Brownian motion. It was realised (most notably by Hunt, in a series of papers in 1957 and 1958 - see Doob (1984) for references) that potential theories could usefully be associated to Markov processes, and much work was done (by Brelot, Deny, and others) on axiomatization and generalization of potential theory. The book Probability and Potential (Meyer (1966)) explored this link, and was later rewritten in a five-volume work of the same title by Dellacherie and Meyer. Soon after Meyer's book, Blumenthal and Getoor (1968) appeared, and was for a long time the standard work on Markov processes and potential theory. Doob's interest in potential theory resulted in his later - and even longer - book Doob (1984), written in his retirement.

The other principal achievement of Meyer and the Strasbourg (or, more generally, French) school has been their 'general theory of processes'. A monograph treatment of Markov process theory, in the light of this general theory of processes, was given by Sharpe (1988). For an insight into the formidable technical problems in this area, see the review Rogers (1989). One service done by the book Protter (1990) was to make available for a wider audience - applied workers, in particular - what the 'probabilist in the street' needs to know about the general theory of processes.

As a glance at the length of the list of books cited here underlines, there will never be another Doob-the-book. If one person deserves to stand comparison with Doob-the-man during the halfcentury discussed here, that person must be Meyer. It is perhaps fair to remark that while the broad thrust of Meyer's work began with Markov processes, it changed towards martingales during his lifetime, and it is here, it seems, that his impact will be deepest.

\section{Post-Doob: the legacy to applied probability}

The viewpoint and results of martingale theory are perhaps the most important of the legacies of Doob's book. Their influence is all around us. In mainstream probability theory see, for example, Rogers and Williams (1994), (1987); in limit theorems see Ethier and Kurtz (1986) (martingale problems) and Jacod and Shiryaev (1987) (convergence of semimartingale characteristics). In analysis, see Durrett (1984). In statistics see, for example, the book of Heyde (1997) on quasi-likelihood, and the extensive literature on sequential analysis. A further area of statistics where martingale methods are crucial is survival analysis and event-history data; see, for example, Andersen et al. (1993).

Within applied probability, one area where martingales have been particularly crucial is queues. Here, interest centres on random discrete times, when customers arrive and depart, etc., and the relevant theory, 'Palm martingale calculus', is a discrete, Poisson-based counterpart to the (harder, better-known and earlier) continuous, Gaussian-based Itô calculus. For textbook expositions see, for example, Brémaud (1981), (1999) and Baccelli and Brémaud (1994).

Rather in the same vein are applications to point processes. Here the main theme is intensity, i.e. the propensity of what can happen, to happen; typical things that can happen here include earthquakes and volcanic eruptions. For background see, for example, Daley and Vere-Jones (1988) - in particular, Chapters 12 and 13.

Branching processes provide another rich field of application. See, for example, Athreya and Ney (1972), Jagers (1975), Lynch (2000), and references therein, and many papers by 
J. D. Biggins. Similarly for other population models such as birth-and-death processes, and within mathematical biology more generally.

The theory of collective risk has been central in applied probability since the early work of Cramér. Random walk methods have long been used here - see, for example, Feller (1966). Martingale methods were fruitfully introduced into the area of insurance and actuarial mathematics in the 1970s by H. U. Gerber; see, for example, Gerber (1986).

Finally, following the work of Black and Scholes (1973), the field of mathematical finance has been transformed by the introduction of relevant stochastic methods; for background and references, we refer the reader to Bingham and Kiesel (2004). First, Itô calculus was introduced into the field by Merton as early as 1973, and then martingale methods were introduced by Harrison and Pliska (1981). The crux of the subject are equivalent martingale measures: probability measures, equivalent to the original one, under which discounted prices become martingales.

\section{Postscript}

The name Doob is so famous, and so unusual, that its origins may be worth recording here, for historical interest. Doob's father was Czech, and 'dub' in Czech means 'oak'. (Readers may recall Alexander Dubček and the Prague Spring of 1968. 'Dubček' means 'little oak' to the delight of the cartoonists of that time.) When Doob's father, then Dub, emigrated to the USA, he got sick of being called 'dub', and changed his name to Doob. To change the spelling to preserve the pronunciation is rare. Usually, the spelling is preserved and the pronunciation changed (thus the distinguished probabilist and statistician Jack Wolfowitz, father of the US Deputy Secretary of Defence, chose to be 'wolf-oh-wits'; similarly for Wiener, etc.).

\section{Acknowledgements}

This paper was written during a visit to the Australian National University in July 2003. I thank the Centre for Mathematics and Its Applications and the Mathematical Sciences Institute at ANU, and colleagues there, for their help and hospitality.

\section{References}

Andersen, P. K., Borgan, Ø., Gill, R. D. And Keiding, N. (1993). Statistical Models Based on Counting Processes. Springer, New York.

Athreya, K. B. And Ney, P. E. (1972). Branching Processes. Springer, New York.

Baccelli, F. And Brémaud, P. (1994). Elements of Queueing Theory. Palm-Martingale Calculus and Stochastic Recurrences (Appl. Math. (New York) 26). Springer, Berlin.

Bartlett, M. S. (1955). Stochastic Processes, with Special Reference to Methods and Applications. Cambridge University Press.

Bertoin, J. (1996). Lévy Processes (Camb. Tracts Math. 121). Cambridge University Press.

Billingsley, P. (1979). Probability and Measure. John Wiley, New York.

Bingham, N. H. (2000). Studies in the history of probability and statistics. XLVI. Measure into probability: from Lebesgue to Kolmogorov. Biometrika 87, 145-156.

Bingham, N. H. And Kiesel, R. (2004). Risk-Neutral Valuation. Pricing and Hedging of Financial Derivatives, 2nd edn. Springer, London.

Blumenthal, R. M. And Getoor, R. K. (1968). Markov Processes and Potential Theory. Academic Press, New York. Breiman, L. (1968). Probability. Addison-Wesley, Reading, MA.

Brémaud, P. (1981). Point Processes and Queues. Martingale Dynamics. Springer, New York.

Brémaud, P. (1999). Markov Chains: Gibbs Fields, Monte Carlo Simulation, and Queues. Springer, New York.

Brockwell, P. J. ANd Davis, R. A. (1987). Time Series: Theory and methods. Springer, New York.

Chung, K.-L. (1960). Markov Chains with Stationary Transition Probabilities. Springer, New York.

Chung, K.-L. (1968). A Course in Probability Theory. Academic Press, Reading, MA. 
Cohn, H. (ed.) (1993). Doeblin and Modern Probability. American Mathematical Society, Providence, RI.

Cramér, H. (1937). Random Variables and Probability Distributions (Camb. Tracts Math. 36). Cambridge University Press.

Cramér, H. (1976). Half a century with probability. Some personal recollections. Ann. Prob. 4, 509-546.

Daley, D. J. And Vere-Jones, D. (1988). An Introduction to the Theory of Point Processes. Springer, New York.

Davis, M. H. A. (1977). Linear Estimation and Stochastic Control. Chapman and Hall, London.

Doob, J. L. (1949). Time series and harmonic analysis. In Proc. Berkeley Symp. Math. Statist. Prob., University of California Press, Berkeley, CA, pp. 303-343.

Doob, J. L. (1953). Stochastic Processes. John Wiley, New York.

Doob, J. L. (1984). Classical Potential Theory and Its Probabilistic Counterpart (Fundamental Principles Math. Sci. 262). Springer, New York.

Doob, J. L. (1990). Review of Masani (1990). Bull. London Math. Soc. 22, 621-623.

Durrett, R. (1984). Brownian Motion and Martingales in Analysis. Wadsworth, Belmont, CA.

Dynkin, E. B. (1965). Markov Processes, Vols 1, 2. Springer, Heidelberg.

Ethier, S. N. And Kurtz, T. G. (1986). Markov Processes. Characterization and Convergence. John Wiley, New York. Feller, W. (1950). An Introduction to Probability Theory and Its Applications, Vol. 1. John Wiley, New York.

Feller, W. (1966). An Introduction to Probability Theory and Its Applications, Vol. 2. John Wiley, New York.

Fernique, X. (1975). Regularité des trajectoires des fonctions aléatoires gaussiennes. In Ecole d'Eté de Probabilités de Saint-Flour, IV-1974 (Lecture Notes Math. 480), Springer, Berlin, pp. 1-96.

GeIrInger, H. (ed.) (1964). Richard von Mises. Mathematical Theory of Probability and Statistics. Academic Press, New York.

GERBER, H. U. (1986). Lebensversicherungsmathematik. Springer, Berlin.

Gīhman, İ. İ. AND SKorokhod, A. V. (1969). Introduction to the Theory of Random Processes. Saunders, Philadelphia, PA.

Gīmman, Ĭ. İ. And Skorokhod, A. V. (1974). The Theory of Stochastic Processes, Vol. I. Springer, New York.

Gīhman, İ. İ. AND SKorokHod, A. V. (1975). The Theory of Stochastic Processes, Vol. II. Springer, New York.

Gīmman, İ. İ. And SKorokHod, A. V. (1979). The Theory of Stochastic Processes, Vol. III. Springer, New York.

Gnedenko, B. V. And Kolmogorov, A. N. (1954). Limit Theorems for Sums of Independent Random Variables. Addison-Wesley, Reading, MA.

Grimmett, G. R. and Stirzaker, D. (1982). Probability and Random Processes. Oxford University Press.

Hall, P. G. ANd Heyde, C. C. (1980). Martingale Limit Theory and Its Applications. Academic Press, Reading, MA. Halmos, P. R. (1950). Measure Theory. Van Nostrand, New York.

Harrison, J. M. AND Pliska, S. R. (1981). Martingales and arbitrage in multi-period securities markets. Stoch. Process. Appl. 11, 215-260.

Heyde, C. C. (1997). Quasi-Likelihood and Its Applications. A General Approach to Optimal Parameter Estimation. Springer, New York.

Ibragimov, I. A. And Rozanov, Yu. A. (1978). Gaussian Random Processes. Springer, New York.

Ikeda, N. And Watanabe, S. (1981). Stochastic Differential Equations and Diffusion Processes. North-Holland, Amsterdam.

Itô, K. And McKean, H. P. (1965). Diffusion Processes and Their Sample Paths. Springer, New York.

JACOD, J. And Shiryaev, A. N. (1987). Limit Theorems for Stochastic Processes. Springer, Berlin.

JAGERS, P. (1975). Branching Processes with Biological Applications. John Wiley, London.

Janson, S. (1997). Gaussian Hilbert Spaces (Camb. Tracts Math. 129). Cambridge University Press.

Kaczmarz, S. and Steinhaus, H. (1935). Theorie der Orthogonalreihen. Monografje Matematyczne, Warsaw.

Kahane, J.-P. (1985). Some Random Series of Functions, 2nd edn. Cambridge University Press.

Kakutani, S. (1944). Two-dimensional Brownian motion and harmonic functions. Proc. Imp. Acad. Tokyo 20, 648-652.

Karatzas, I. and Shreve, S. E. (1988). Brownian Motion and Stochastic Calculus. Springer, New York.

Karlin, S. And Taylor, H. (1975). A First Course in Stochastic Processes. Academic Press, Reading, MA.

Karlin, S. and Taylor, H. (1981). A Second Course in Stochastic Processes. Academic Press, Reading, MA.

Kingman, J. F. C. AND TAYlor, S. J. (1966). An Introduction to Measure and Probability. Cambridge University Press.

Kolmogorov, A. N. (1933). Grundbegriffe der Wahrscheinlichkeitsrechnung. Springer, Berlin.

Krengel, U. (1985). Ergodic Theorems. De Gruyter, Berlin.

LÉvy, P. (1925). Calcul des Probabilités. Gauthier-Villars, Paris.

LÉvy, P. (1937). Théorie de l'addition des Variables Aléatoires. Gauthier-Villars, Paris.

LÉvy, P. (1948). Processus Stochastiques et Mouvement Brownien. Gauthier-Villars, Paris.

Lindvall, T. (1991). W. Doeblin (1915-1940). Ann. Prob. 19, 929-934.

LoÈve, M. (1955). Probability Theory. Van Nostrand, New York.

LoÈve, M. (1973). Obituary: Paul Lévy, 1886-1971. Ann. Prob. 1, 1-18.

LoÈve, M. (1977). Probability Theory. I., 4th edn. Springer, New York. 
LoÈve, M. (1978). Probability Theory. II., 4th edn. Springer, New York.

LYNCH, J. D. (2000). The Galton-Watson process revisited: some martingale relationships and applications. J. Appl. Prob. 37, 322-328.

Lyons, T. J. AND Qian, Z. (2002). System Control and Rough Paths. Oxford University Press.

McKean, H. P. (1969). Stochastic Integrals. Academic Press, New York.

Masani, P. R. (1990). Norbert Wiener. Birkhäuser, Basel.

Meyer, P.-A. (1966). Probability and Potentials. Blaisdell, Waltham, MA.

Meyer, P.-A. (1976). Un cours sur les intégrales stochastiques. In Séminaire de Probabilités (Lecture Notes Math. 511), Vol. X, Springer, Heidelberg, pp. 245-398.

Meyn, S. P. AND Tweedie, R. L. (1993). Markov Chains and Stochastic Stability. Springer, New York.

Neveu, J. (1975). Discrete-Parameter Martingales. North-Holland, Amsterdam.

Nummelin, E. (1984). General Irreducible Markov Chains and Nonnegative Operators. Cambridge University Press.

ØKsendal, B. (1985). Stochastic Differential Equations. An Introduction with Applications. Springer, New York.

Protter, P. (1990). Stochastic Integration and Differential Equations. A New Approach (Appl. Math. (New York) 27). Springer, New York.

RÉvÉsz, P. (1968). The Laws of Large Numbers. Academic Press, New York.

Revuz, D. (1984). Markov Chains, 2nd edn. North-Holland, Amsterdam.

Revuz, D. ANd Yor, M. (1991). Continuous Martingales and Brownian Motion. Springer, Berlin.

Rogers, L. C. G. (1989). Review of Sharpe (1988). Bull. London Math. Soc. 21, 622-623.

Rogers, L. C. G. ANd Williams, D. (1994). Diffusions, Markov Processes and Martingales, Vol. 1, Foundations. John Wiley, Chichester.

Rogers, L. C. G. ANd Williams, D. (1987). Diffusions, Markov Processes and Martingales, Vol. 2, Itô Calculus. John Wiley, Chichester.

Sato, K.-I. (1999). Infinite Divisibility and Lévy Processes. Cambridge University Press.

Sharpe, M. J. (1988). General Theory of Markov Processes. John Wiley, New York.

SPITZER, F. (1964). Principles of Random Walk. Van Nostrand, Princeton, NJ.

Stone, M. H. (1932). Linear Transformations in Hilbert Space and Their Applications to Analysis (AMS Colloq. Pub. 15). American Mathematical Society, Providence, RI.

Stroock, D. W. and Varadhan, S. R. S. (1979). Multidimensional Diffusions. Springer, New York.

Whittle, P. (1963). Prediction and Regulation by Linear Least-Squares Methods. English Universities Press, London. WIENER, N. (1949). Extrapolation, Interpolation and Smoothing of Stationary Time Series. John Wiley, New York.

WiEner, N. (1976). Collected Works, Vol. I. MIT Press, Cambridge, MA.

WiEner, N. (1979). Collected Works with Commentaries, Vol. II. MIT Press, Cambridge, MA.

WiEner, N. (1981). Collected Works with Commentaries, Vol. III. MIT Press, Cambridge, MA.

WiEner, N. (1985). Collected Works with Commentaries, Vol. IV. MIT Press, Cambridge, MA.

Zygmund, A. (1935). Trigonometric Series. Monografje Matematyczne, Warsaw (reprinted: Dover, New York, 1955). 